\title{
Lidil
}

Revue de linguistique et de didactique des langues

\section{Lire des récits au cours préparatoire : une expertise professionnelle contrastée et encore largement à construire}

Reading Narrative in First-grade Classes: A Professional Expertise Still Largely to Be Built

\section{Anne Vadcar et Catherine Frier}

\section{OpenEdition}

Journals

Édition électronique

URL : http://journals.openedition.org/lidil/4156

DOI : 10.4000/lidil.4156

ISSN : $1960-6052$

Éditeur

UGA Éditions/Université Grenoble Alpes

Édition imprimée

ISBN : 978-2-37747-002-0

ISSN : $1146-6480$

\section{Référence électronique}

Anne Vadcar et Catherine Frier, « Lire des récits au cours préparatoire : une expertise professionnelle contrastée et encore largement à construire », Lidil [En ligne], 55 | 2017, mis en ligne le 02 mai 2017, consulté le 04 mai 2019. URL : http://journals.openedition.org/lidil/4156 ; DOI : 10.4000/lidil.4156

Ce document a été généré automatiquement le 4 mai 2019.

(c) Lidil 


\section{Lire des récits au cours} préparatoire : une expertise professionnelle contrastée et encore largement à construire

Reading Narrative in First-grade Classes: A Professional Expertise Still Largely to Be Built

Anne Vadcar et Catherine Frier

\section{Contexte de la recherche, jalons théoriques, objectifs et hypothèses}

\subsection{Une exploration des pratiques des enseignants}

1 Cet article prend sa source dans la recherche IFÉ intitulée «L'influence des pratiques d'enseignement de la lecture et de l'écriture sur la qualité des apprentissages au cours préparatoire " et pilotée par Roland Goigoux. Commencée en 2011, cette recherche explore les pratiques d'enseignement de la lecture et de l'écriture en classe de Cours préparatoire ( $\mathrm{CP}, 1^{\mathrm{re}}$ année primaire) dans une perspective à la fois quantitative et qualitative. Les chercheurs ont enquêté en 2013/2014 dans plus de 130 classes de CP de France métropolitaine, sur un échantillon dit « de bon aloi ».

Plus précisément, ce projet vise à mettre en évidence l'effet des pratiques d'enseignement du lire-écrire sur les apprentissages. Par ce biais, il cherche aussi à éclairer la problématique des inégalités scolaires, problématique qui reste centrale dans le champ de la didactique du français (Nonnon \& Goigoux, 2007). Au sein de ce vaste projet, un groupe de travail s'intéresse plus spécifiquement à la question des pratiques d'acculturation à l'écrit en classe de CP. Ces pratiques, que l'on peut également qualifier d'éducation à la culture de l'écrit, paraissent avoir un effet qui favorise le développement des 
compétences globales en lecture-écriture, en particulier pour les élèves initialement de niveau faible ou intermédiaire. En effet, pour la première fois, une étude scientifique (Tiré, Vadcar, Ragano \& Bazile, 2015) révèle de manière objective l'impact positif des pratiques acculturantes sur la progression globale des élèves. Ces résultats montrent notamment qu'au sein d'un ensemble de pratiques d'acculturation diversifiées, l'utilisation massive du récit est un facteur déterminant ${ }^{1}$.

3 Les prescriptions officielles pour le CP et plus largement pour le cycle 2 imposent d'«Écouter et de lire des œuvres intégrales, notamment de littérature de jeunesse » (socle commun palier 1, 2011), mais aucune précision n'est donnée dans ces programmes en termes d'objectifs quantitatifs autour de la lecture de récits (temps consacré dans l'horaire hebdomadaire par exemple), d'apprentissages précis (compétences visées chez l'élève) ni de critères concernant ce format pédagogique particulier, un peu comme si ce domaine relevait plus d'un consensus mou que d'une véritable volonté didactique associée à un savoir professionnel clairement identifié. Bishop (2012) relève que, si la lecture de récit à l'école maternelle est bien un genre professionnel, ses finalités sont très mouvantes. Depuis quelques années, cette pratique de lecture d'un adulte à l'enfant, qu'elle soit scolaire ou familiale, est devenue un objet de réflexion didactique et une profusion de termes a émergé pour la qualifier. Boiron (2010) parle de «lecture répertoire » qui ne convoque pas d'interactions et qui vise la constitution d'un répertoire d'œuvres partagées, de «lecture médiatisée » qui implique une médiation de l'acte de lecture par le maitre, et enfin de «lecture autonome» menée par le jeune lecteur pour lui-même. Pour Giasson (2011), la « lecture partagée » est proche de la lecture des parents aux enfants et implique qu'on montre le texte en même temps qu'on lit et qu'un temps d'interactions soit ajouté. Le terme de "lecture offerte", apparu dans les programmes de 2016 (une occurrence pour le programme cycle 2), est très répandu dans le vocable utilisé par les enseignants du primaire sans qu'il ait un appui dans la littérature scientifique. De la même manière que la lecture partagée familiale est hétérogène (Bonnéry \& Joigneaux, 2015; Frier, 2006), la lecture offerte scolaire recouvre certainement plusieurs sens pour les maitres de CP.

4 On sait que le récit écrit se distingue du récit oral par un ensemble de caractéristiques spécifiques de l'écrit: syntaxe et lexique choisis (Chauveau, 1997), durée du récit, décontextualisation (Olson, 1999), abstraction du locuteur (Chauveau, 1997), part implicite du message. Les travaux récents des sociologues (Bonnéry, 2012) sur l'usage de l'album de littérature de jeunesse, principal vecteur du récit en classe maternelle, montrent, à travers une approche historique et sociologique, qu'il s'agit d'un genre dont l'appropriation n'a rien d'inné, mais relève au contraire de codes, de savoirs et de savoirfaire (linguistiques, sémiotiques, sociaux, etc.) très spécialisés. Des enquêtes complémentaires (Bonnéry, 2014 ; Bonnéry, 2015) portant sur les usages de la littérature de jeunesse dans les familles et dans l'école mettent en évidence des choix de livres et des manières de lire différentes selon le contexte social des écoles ou des familles. Selon Bonnéry, ces variations curriculaires renvoient aux contextes socio-familiaux des élèves, et ne permettent pas d'enseigner à tous des manières de lire à partir de contenus communs ${ }^{2}$. Autrement dit, pour ces auteurs, les enseignants ont tendance à « inégaliser » leurs pratiques en fonction de ces différences curriculaires, ce qui renforce, de fait, les inégalités scolaires.

5 Les didacticiens se sont intéressés de leur côté à la nature des interactions langagières autour du livre en contexte familial, montrant que la médiation au récit s'inscrit elle aussi 
dans un corps de pratiques ritualisées et construites socialement (Frier, 2006). D'autres travaux portent sur les pratiques enseignantes dans l'apprentissage de la lecture au cycle 2, mais davantage dans la classe de Grande section (GS) que dans la classe de CP (Boiron, 2010 ; Bourhis, 2012 ; Dionne, 2013 ; Grossmann, 1996). Par ailleurs, si nombre de ces travaux ont cherché à décrire les «bonnes pratiques » des enseignants (Nonnon \& Goigoux, 2007), on peut néanmoins remarquer que « paradoxalement, on dispose encore actuellement de peu de descriptions pour établir cet état des lieux» (ibid., p.9) aux différents niveaux de la scolarité primaire. Si le travail réalisé sur les pratiques des maitres de CP par Piquée et Sensevy (2007) apporte un éclairage fort intéressant sur la question, il a été réalisé à partir d'un recueil de données déclaratif (questionnaires) et non d'observations sur le terrain. Ainsi, le modèle scolaire de la lecture de récit, en particulier au $\mathrm{CP}$, semble encore largement à découvrir puisque finalement peu de travaux ont exploré les pratiques réelles des enseignants du $\mathrm{CP}$ dans ce domaine.

\subsection{Les contours d'un rituel instituant le jeune lecteur}

6 La lecture d'un adulte faite à l'enfant, que ce soit dans l'intimité de la famille ou la collectivité de la classe peut être considérée comme un rituel. Le rituel n'est pas défini de manière fixe dans la littérature, cependant, la définition proposée par Piette (1997, p. 150), est assez large pour faire percevoir en quoi la lecture magistrale est rituelle. Selon cet auteur, il s'agit d'un "ensemble d'actions, insérées dans un contexte spécifique ludico-fictionnel, dont le déroulement et la perception cognitive et/ou émotionnelle se font selon quatre formes non exclusives (cérémonie, compétition, fête et spectacle)». De plus, comme le rappelle Merri et Vannier (2015), le rituel « fait référence, [...], à des actes qui sacralisent le social (Javeau, 2001), tel le gâteau d'anniversaire» (p. 3). Le rituel possède les caractéristiques qui favorisent le passage d'un état à un autre au sein d'une phase liminale (Turner, 1969) et permet ainsi d'être un acte d'institution (Bourdieu, 1982), créateur à la fois d'identité et d'unité, c'est-à-dire qui institue une communauté faite d'individualités. La lecture du soir ou la lecture offerte par l'enseignant à ses élèves sont donc des espaces au sein desquels l'enfant peut forger une identité de lecteur et intégrer la communauté des lecteurs. Nous nous sommes appuyées sur la description du rituel de Wulf et coll. (2005) pour mener notre analyse :

Les rituels sont des mises en scène sociales vécues par les sens dans lesquelles surgit une expérience de la différence. Les rituels sont des représentations culturelles, et, en tant que telles, sont corporels, performatifs, expressifs, symboliques, régularisés, non instrumentaux et efficients. [...] Les rituels sont des modèles institutionnels de mises en scène du savoir collectif des pratiques d'actions communes qui permettent de confirmer l'autoreprésentation et l'autointerprétation de l'ordre institutionnel ou communautaire. Leur arrangement scénique comporte des facteurs de reproduction, de construction et d'innovation. (p. 8)

Le rituel de lecture de l'adulte à l'enfant est ainsi la mise en scène d'un savoir pratique dans lequel les différents acteurs, dont le livre, ont un rôle à jouer dans un cadre spatiotemporel où la gestualité et les paroles instituent les rôles et où les relations de pouvoir sont susceptibles d'être modifiées. Nous faisons l'hypothèse que ce rituel permet de représenter la communauté des lecteurs à travers la pratique de la lecture privée de littérature. Par ailleurs, le caractère performatif du rituel ne garantit pas le but poursuivi. Wulf et coll. (2005) précisent que "Même si plusieurs actions se basent sur la même intention, elles se différencient dans la manière d'être accomplie » (p. 405). Ainsi le rituel 
de lecture magistrale peut prendre un sens différent pour les enfants simplement dans la manière dont il est accompli par l'enseignant ou le parent.

\subsection{Objectifs et hypothèses}

8 Rappelons que l'objectif de cet article est de décrire et d'analyser le modèle scolaire de la lecture de récit au $\mathrm{CP}$, modèle encore très largement à découvrir. Nous allons dans ce texte tenter de répondre à ces différentes questions: Les maitres de CP lisent-ils des récits à leurs élèves ? À quelle fréquence ? Et de quelle(s) manière(s) ? La lecture de récit au CP est-elle une pratique «naturelle » chez la plupart des enseignants ou au contraire un savoir-faire professionnel hétérogène encore largement à construire? Quelles formes prend ce rituel? Peut-on identifier un savoir-faire différencié selon la zone d'éducation prioritaire ou non?

9 Pour répondre à ces questions, nous allons explorer à la fois les choix didactiques et pédagogiques ${ }^{3}$ des enseignants.

Notre but est donc d'étudier cette médiation au récit écrit en tant que compétence professionnelle à part entière de l'agir enseignant (Jorro, 2007), compétence dont on a souvent du mal à saisir l'importance, surtout pour les élèves dont la fréquentation familiale de la culture écrite et du récit en particulier est faible. Le contexte scolaire, qui se démarque si fortement du contexte familial, se doit donc «de penser en permanence les articulations qui permettront à la lecture collective de déboucher sur une activité subjective » (Dezutter \& Dufays, 2015, p. 8). Dans cette perspective, le rôle, très complexe, du maitre, consiste à mettre en place un contexte d'apprentissage qui est d'autant plus efficace qu'il permet à un plus grand nombre d'élèves de s'y inscrire (Nonnon \& Goigoux, 2007). Par contexte d'apprentissage ou "gestes professionnels ", nous entendons à la fois les choix didactiques et pédagogiques effectués par le maitre, sans émettre d'hypothèse à priori sur le poids respectif de ces différents choix sur la progression des élèves.

11 Nous faisons l'hypothèse que cette médiation au récit au $\mathrm{CP}$ :

- relève d'une expertise professionnelle qui se décline à travers des gestes précis restant à qualifier;

- s'inscrit dans le cadre d'une ritualisation à l'interface de la sphère scolaire, sociale et culturelle ;

- n'est pas homogène et peut prendre des formes différentes selon le niveau d'expertise de l'enseignant dans ce domaine ;

- a un impact sur la construction du rapport à la lecture des élèves et de leur compétence de lecteurs en devenir, que ce soit en ZEP ou hors ZEP.

\section{La démarche méthodologique mise en œuvre}

12 L'étude s'appuie sur un échantillon de classes du corpus de la recherche Lire-Écrire au CP pratiquant la lecture offerte (désormais LO). Comme l'indique son intitulé, cette recherche s'intéresse à "L'influence des pratiques d'enseignement de la lecture et de l'écriture sur la qualité des apprentissages au cours préparatoire ». Aussi, elle comporte un volet "évaluation" qui a permis de mesurer "toutes choses égales par ailleurs " l'effet qu'on peut attribuer aux pratiques de l'enseignant sur les performances des élèves dans le domaine du lire-écrire. Les 131 classes ont ainsi pu être classées selon le degré 
d'influence positive des pratiques de l'enseignant, des plus au moins performantes. À la suite de ce résultat et dans une perspective contrastive, nous avons constitué deux échantillons parmi les classes les plus et les moins performantes de la recherche LireÉcrire au CP, c'est-à-dire celles pour lesquelles l'« effet-maitre » est fort ou, au contraire, faible. Les classes ont été sélectionnées à la suite d'un premier repérage effectué à partir des réponses au questionnaire interrogeant les enseignants sur l'utilisation d'albums avec leurs élèves. Un ensemble de classes dans lesquelles des récits étaient lus en lecture magistrale a ainsi été identifié. Nous nous sommes ensuite appuyées sur les résultats de Lire-Écrire au CP pour constituer les deux échantillons. Huit classes, dont les vidéos permettaient une analyse adéquate, ont été retenues parmi les plus performantes (échantillon nommé désormais Class+) et sept parmi les moins performantes (désormais Class-). Ces échantillons contiennent des classes en éducation prioritaire, deux pour Class + et une pour Class- et correspondent de ce point de vue à la répartition nationale. Selon les classes, une ou plusieurs séances de LO ont été retenues sur les semaines 47, 12 et 21 de l'année scolaire.

Notre objectif étant d'identifier les pratiques réelles, nous avons travaillé sur les vidéos des séances de LO qui sont la base de notre étude. Il est important de noter que ces séances correspondent à des moments ordinaires de la classe, conformément à la méthodologie retenue pour la recherche Lire-Écrire au $C P^{4}$.

L'observation a été menée par les deux auteures à l'aide d'un tableau de critères portant sur :

- la durée de la séance (totale, temps de lecture, temps d'interaction avant et après la lecture);

- le support de lecture (album lu);

- les formes et le degré de ritualisation (gestuelle, lieu et configuration de l'espace de LO, déroulement général de la séance, alternance et contenu des moments identifiables);

- la qualité de la lecture oralisée par le maitre (vitesse de diction, prosodie, régulation de l'interaction texte entendu/élève);

- la qualité de l'interaction maitre-élève (forme, contenu et orientations des interactions maitre-élèves à propos du texte, type de participation des élèves attendue par le maitre, régulation et étayage des interventions);

- l'investissement du maitre dans la tâche de LO (aspects gestuels et proxémiques, regards, prosodie, gestion de l'attention conjointe, tissage avec d'autres tâches);

- le type d'investissement des élèves pendant la LO et les temps d'interaction (degré d'attention conjointe, posture corporelle, type d'oral mobilisé).

Cette observation peut être qualifiée d'observation assistée par vidéo. Cette méthode, qui est utilisée aussi en ethnologie, permet de se positionner à la fois en tant qu'observateur et participant. L'observation n'est cependant que périphérique car nous n'étions pas dans la classe et n'avons joué aucun rôle dans le déroulement des tâches. Lors de l'observation des vidéos de lectures, nous avons adopté dans un premier temps le point de vue de l'élève pour ressentir l'effet produit par la lecture du maitre; tout comme eux, nous étions spectateurs et auditeurs. Ce n'est que dans un second temps que nous nous sommes attachées à quantifier et qualifier le travail du maitre, puis à décrire la manière dont il met en œuvre ce moment. Le comportement des élèves a donc été pris en compte dans cette optique, c'est-à-dire en relation avec la situation installée par le maitre, mais nous n'avons pas cherché à identifier individuellement les apprentissages potentiellement effectués. 


\section{Présentation des résultats}

\subsection{Des résultats quantitatifs qui mettent en évidence un premier niveau de contraste entre Class+ et Class-}

\subsubsection{Inscription de la pratique de LO dans la durée}

16 Bien que la LO soit une pratique généralisée dans les classes de CP (90,84\% des classes de Lire-Écrire au CP), elle n'est néanmoins pas constante sur l'année : $65 \%$ des classes l'ont déclarée au moins sur deux des trois semaines de l'enquête. Dans nos échantillons Class+ et Class-, cette différence est accentuée. $75 \%$ de Class+ en font une pratique régulière tout au long de l'année contre $57 \%$ de Class-.

\subsubsection{Un format de séance plus ou moins stabilisé}

17 On observe des séances en un, deux ou trois temps, parmi lesquels on distingue deux temps consacrés exclusivement à des interactions (temps a et c) qui soit préparent à la lecture (a), soit sont le prolongement de la lecture (c), et un temps pendant lequel le texte est lu (temps b).

L'usage des formats et les durées accordées à ces trois temps sont très différents entre Class+ et Class-.

Tableau 1. - Format des séances de LO.

\begin{tabular}{|l|l|l|l|l|l|l|}
\hline & \multicolumn{4}{|l|}{ Format de la séance } & \multicolumn{2}{l|}{$\begin{array}{l}\text { Part respective des durées de lecture et } \\
\text { d'interactions sur la séance de LO }\end{array}$} \\
\hline $\begin{array}{l}3 \text { temps } \\
(\mathrm{a}, \mathrm{b}, \mathrm{c})\end{array}$ & $\begin{array}{l}2 \text { temps } \\
(\mathrm{a}, \mathrm{b})\end{array}$ & $\begin{array}{l}2 \text { temps } \\
(\mathrm{b}, \mathrm{c})\end{array}$ & $\begin{array}{l}1 \text { temps } \\
(\mathrm{b})\end{array}$ & Lecture (b) & $\begin{array}{l}\text { Interactions } \\
(\mathrm{a}, \mathrm{c})\end{array}$ \\
\hline Class+ & $72 \%$ & $17 \%$ & $6 \%$ & $6 \%$ & $58 \%$ & $42 \%$ \\
\hline Class- & $40 \%$ & $30 \%$ & $10 \%$ & $20 \%$ & $73 \%$ & $27 \%$ \\
\hline
\end{tabular}

Le format de séance en trois temps est la norme dans Class+; à l'inverse, dans Class-, le format en trois temps a autant de place que les formats en deux temps et la part des séances sans interactions est plus importante. On observe simultanément que la proportion accordée au temps de lecture est bien plus importante dans Class- que dans Class+ ( $73 \%$ contre $58 \%$ ). Les vidéos révèlent deux types de scénarios pour ce temps de lecture du texte. Soit le texte est lu intégralement avec très peu d'interventions du maitre, soit les interactions maitre-élèves sont intensément mêlées au texte lu. La grande part accordée au temps de lecture dans Class- est le fait tantôt d'une lecture qui est émaillée de questions et de commentaires, tantôt d'une faible importance accordée aux interactions après lecture. Dans Class+, pendant le temps consacré à la lecture, le maitre intervient uniquement à la marge et de manière laconique pour remédier à une difficulté de lexique ou rendre vraiment saillant un moment clé du récit. Les temps d'interactions après lecture ont en revanche une place importante dans la séance. 


\subsubsection{Un degré d'enrôlement dans la tâche variable}

\subsubsection{Le cadre spatial, de l'usage scolaire à l'usage social} classe dans laquelle ils ont été filmés. Ils ont organisé l'espace en cohérence avec leur pratique. On observe ainsi des coins lecture avec une bibliothèque et des coins regroupement équipés. Certains enseignants privilégient aussi l'installation aux bureaux en toute situation et font ou non varier l'orientation des élèves vers le tableau. La plupart des enseignants (un seul de Class- reste debout) adoptent une posture assise qui contextualise l'acte de lecture. Le tableau rend compte de ces choix dans les échantillons Class+ et Class- lors des séances de LO.

Tableau 2. - Organisation spatiale des séances de LO.

\begin{tabular}{|l|l|l|l|l|l|}
\hline & \multicolumn{2}{|l}{ Lieu de la séance } & \multicolumn{2}{l|}{$\begin{array}{l}\text { Disposition } \\
\text { élèves }\end{array}$} \\
\hline & Coin lecture & Coin regroupement & Classe & Face tableau & Autre \\
\hline Class+ & $50 \%$ & $37 \%$ & $13 \%$ & $0 \%$ & $100 \%$ \\
\hline Class- & $0 \%$ & $43 \%$ & $57 \%$ & $71 \%$ & $29 \%$ \\
\hline
\end{tabular}

Le cadre spatial réservé aux séances de LO peut être rangé sur un axe allant de la lecture privée à la lecture scolaire. De l'utilisation d'un coin lecture à la configuration purement scolaire, les enseignants jouent avec les possibilités matérielles offertes et leurs représentations de la LO. Dans Class+, les séances de LO se déroulent majoritairement dans une organisation spatiale particulière où les élèves se retrouvent groupés; à l'inverse, dans Class-, l'organisation spatiale scolaire est privilégiée : les enfants sont à leur table de travail face au tableau et l'enseignant se situe entre eux et le tableau.

\subsubsection{La gestualité du maitre : un rôle médiateur entre l'élève lecteur et le texte}

La gestualité de l'enseignant est également signifiante. En effet, les gestes et les mimiques représentent de manière symbolique des intentions et des émotions et participent de ce fait à la socialisation de l'individu en dépassant la compréhension explicite et rationnelle (Wulf et coll., 2004, p. 408). Nous avons donc visionné les séances de LO sans le son afin de concentrer notre regard sur la gestualité du maitre et d'identifier des gestes permettant de symboliser le début et la fin de la lecture magistrale, des gestes de pointage vers le livre et les élèves et de qualifier la manipulation du support du texte par l'enseignant ${ }^{5}$.

Tableau 3. - Gestualité du maitre pendant les séances de LO.

\begin{tabular}{|l|l|l|l|}
\hline & & Class+ & Class- \\
\hline Geste de début de lecture & $100 \%$ & $0 \%$ \\
\hline
\end{tabular}




\begin{tabular}{|l|l|l|}
\hline Geste de fin de lecture & $100 \%$ & $0 \%$ \\
\hline Gestes de pointage vers le livre & $100 \%$ & $25 \%$ \\
\hline Gestes de pointage vers les élèves & $100 \%$ & $25 \%$ \\
\hline Manipulation du livre & $\begin{array}{l}\text { Respectueuse et } \\
\text { distanciée }\end{array}$ & $\begin{array}{l}\text { Non distanciée ou } \\
\text { excessivement distanciée }\end{array}$ \\
\hline
\end{tabular}

La comparaison des gestuelles des enseignants de Class+ et Class- est frappante. Tous les enseignants observés de Class+ manifestent d'une manière ou d'une autre l'entrée dans la lecture et sa sortie, mais aucun de ceux de Class- ne le font. La manipulation du livre est elle aussi contrastée entre Class+ et Class-. On observe dans Class+ des gestes respectueux, presque délicats, envers le livre en même temps qu'une attitude plutôt distanciée, alors que, dans Class-, soit la manipulation est très affective (le livre est pressé contre soi de façon un peu fébrile), soit le livre est tenu à distance (à bout de bras) avec excès. Durant les interactions, le livre et les élèves sont l'objet de gestes de pointage dans Class+ mais très peu dans Class-.

\subsubsection{La lecture du maitre à voix haute : de la mise en scène intentionnelle à la mise en scène affective}

Comme l'a montré Boiron (2010), tous les enseignants font une lecture interprétée du texte. En tant que lecteurs experts, ils ont automatisé les processus interprétatifs et la lecture qu'ils livrent aux élèves est déjà une version différente de celle que l'auditeur aurait $\mathrm{pu}$ faire individuellement. La compréhension des élèves résulte donc de l'interaction entre leurs procédures personnelles et la lecture du maitre. À la suite de Bourhis (2012), nous avons, dans nos observations, qualifié de faible à forte la mise en scène affective (basée sur les variations intonatives) et la mise en scène intentionnelle du texte (basée sur l'utilisation de pauses prosodiques longues qui créent des espaces dialogiques entre l'auditeur et le texte lu). Le tableau suivant montre la répartition des mises en scène de toutes les lectures observées.

Tableau 4. - Mises en scène du texte.

\begin{tabular}{|l|l|l|l|l|}
\hline \multicolumn{4}{|l|}{ Class +} & \multicolumn{2}{l|}{ Class- } \\
\hline & \multicolumn{2}{l|}{ Mise en scène } \\
\hline & affective & intentionnelle & affective & intentionnelle \\
\hline faible & $0 \%$ & $0 \%$ & $11 \%$ & $56 \%$ \\
\hline moyenne & $47 \%$ & $16 \%$ & $11 \%$ & $11 \%$ \\
\hline forte & $53 \%$ & $84 \%$ & $78 \%$ & $33 \%$ \\
\hline
\end{tabular}


La mise en scène intentionnelle est beaucoup plus utilisée, de moyennement à fortement, dans l'échantillon Class+, alors que, dans Class-, on ne peut pas l'identifier dans $56 \%$ des lectures observées. À l'inverse, la mise en scène affective est beaucoup plus forte dans l'échantillon Class- que dans Class+. Nous faisons l'hypothèse que les maitres de Class+ parviennent mieux que ceux de Class- à utiliser une mise en scène intentionnelle ce qui les dégage de la nécessité de mettre fortement en scène les affects des personnages et laisse plus de place à l'élève pour intervenir individuellement dans le jeu dialogique auquel le texte invite.

\subsubsection{Des interactions maitre/élèves différemment focalisées}

Dans toutes les LO observées, sauf deux de Class-, le maitre et les élèves interagissent à propos de la lecture. Nous nous sommes attachées à identifier sur quoi sont focalisées ces interactions et selon quelle logique elles sont guidées par l'enseignant. On observe huit objets de dialogues à propos des textes et trois types de logiques qui guident ces échanges.

Tableau 5. - Sujets de discussions à propos des textes.

\begin{tabular}{|l|l|l|}
\hline & Class+ & Class- \\
\hline Thème & $22,2 \%$ & $10,0 \%$ \\
\hline Déroulé de l'histoire & $72,2 \%$ & $10,0 \%$ \\
\hline Intention personnage & $72,2 \%$ & $10,0 \%$ \\
\hline Structure récit & $0,0 \%$ & $30,0 \%$ \\
\hline Liste des personnages & $0,0 \%$ & $20,0 \%$ \\
\hline Vocabulaire & $22,2 \%$ & $30,0 \%$ \\
\hline Intertextualité & $0,0 \%$ & $20,0 \%$ \\
\hline Intérêt pour le texte & $5,6 \%$ & $0,0 \%$ \\
\hline
\end{tabular}

Tableau 6. - Guidage des interactions maitre/élève.

\begin{tabular}{|l|l|l|l|}
\hline & \multicolumn{3}{|l|}{ Guidage des interactions } \\
\hline & Plan & $\begin{array}{l}\text { Contenu à } \\
\text { transmettre }\end{array}$ & Informel \\
\hline Class+ & $100 \%$ & $0 \%$ & $0 \%$ \\
\hline Class- & $0 \%$ & $50 \%$ & $30 \%$ \\
\hline
\end{tabular}


Les enseignants de Class+ permettent presque toujours aux élèves d'exercer leur compétence narrative en les aidant à reconstruire oralement le schéma narratif du récit. Ils étayent également la prise d'indice pour amener les élèves à expliciter les intentions des personnages. Pour cela, ils guident la discussion de manière planifiée en suivant l'ordre du récit. Les enseignants de Class- abordent ces sujets seulement à la marge et se focalisent plutôt sur des aspects littéraires formels tel que la reconnaissance d'une structure comme dans le conte de randonnée ou celle d'un genre (conte ou pas conte). Ils apportent également une plus grande importance au vocabulaire. La discussion est souvent informelle et ouverte ou alors les échanges sont guidés par une logique de transmission de savoirs. Dans ce cas, la discussion est principalement axée sur des aspects formels ou lexicaux et l'enseignant apporte beaucoup d'informations sous la forme de définitions de mots ou d'explications en relation avec la structure du texte.

La comparaison quantitative des pratiques de LO entre Class+ et Class- dessine deux types de profils extrêmes :

- du côté de Class+, un enseignant qui centre sa pratique sur les procédures du lecteur, un usage social de la lecture, et qui a développé une expertise dans les gestes professionnels associés ;

- du côté de Class-, un enseignant qui centre sa pratique sur un usage scolaire de la lecture et des connaissances savantes sur le texte. Les gestes professionnels observés sont hétérogènes et non stabilisés.

29 Essayons à présent d'illustrer ces premiers résultats quantitatifs par des observations plus fines dans deux classes contrastées.

\subsection{Un exemple contrasté d'utilisation du corps enseignant dans la ritualisation de la lecture partagée}

\subsubsection{Contexte}

Dans la classe 61 (Class+), l'enseignant (c61, M61) s'apprête à lire C'était un loup si bête tiré d'un recueil, et dans la classe 54 (Class- (c54, M54), il s'agit de La moufle de Franquin. Dans ces deux classes, les enfants sont réunis dans un espace dans lequel ils suivent habituellement des activités collectives.

\subsubsection{Un positionnement du livre, du maitre et des élèves qui n'a pas les mêmes effets}

Le coin regroupement de M61 est constitué d'un pan de mur contre lequel la chaise basse du maitre est installée et de trois bancs disposés en carré qui clôturent l'espace, le maitre fait face aux élèves qui l'entourent. Avec M54, le regroupement se fait juste devant le tableau, les enfants sont assis sur le sol face au tableau. La maitresse est assise, elle aussi, sur une petite chaise qu'elle a installée sur le côté pour que tous les enfants voient bien le tableau.

Au début de la séance, M61 est assis sur sa chaise et tient entre ses mains le livre qu'il ne montre pas ; M54 est également assise mais ne fait pas face aux élèves, elle est sur le côté et l'album La moufle est exposé sur le porte-craie du tableau. Le spectacle qui s'offre aux élèves est donc bien différent. Dans la c61, ces derniers observent leur maitre qui s'apprête à lire, mais les élèves de c54 ont face à eux un tableau sur lequel est déjà écrit le 
texte de la séance de lecture qui suivra la LO et un album exposé. Les réactions des élèves sont en relation. Ceux de c61 sont silencieux, ils attendent le début, tandis que ceux de c54 commencent à décoder ce qu'ils voient au tableau, dont le titre de l'album. Un élève demande " pourquoi / c'est écrit la moufle ». Pour les élèves de c54, il y a un brouillage du sens de la situation: est-ce une séance de LO, de lecture collective? Quel est le centre d'intérêt de la séance, la maitresse qui lit, le texte du tableau ou bien l'album posé là ? Le choix de l'installation des élèves mais aussi du maitre s'avère ainsi un élément qui oriente l'attention des élèves vis-à-vis de ce qui va suivre et leur fournit des indices sur l'attitude à adopter.

\subsubsection{Entrée et sortie de lecture : I'initiation à une temporalité du lecteur}

33 Au moment de commencer la lecture, M61 lève le livre pour le montrer à tous. Comme il s'agit d'un recueil, il a mis un marque-page qu'on voit dépasser, puis il rabaisse le livre vers ses genoux, ouvre le livre et stocke son marque-page au début puis donne encore quelques consignes aux élèves tout en caressant la première page du conte qu'il va lire. Enfin, il recule légèrement sur sa chaise pour être installé plus confortablement, ses épaules s'abaissent, il pose le doigt sur les lèvres pour signifier le silence et commence à lire. Le début de lecture dans c54 est très différent, il est précédé d'un long échange sur la différence entre moufle et gant au cours duquel M54 ne touche pas le livre et utilise le tableau pour appuyer ses explications avec un dessin. Puis elle efface son dessin à la brosse et pose la main sur le livre exposé, prête à le prendre, patiente encore quelques secondes pour gronder les élèves qui n'ont peut-être pas compris ce qui attendu d'eux. Enfin, elle prend le livre à deux mains et le rapproche d'elle en regardant la couverture, elle ouvre rapidement l'album au début de l'histoire et lève le livre sur sa droite à hauteur de son visage en montrant la double page en même temps qu'elle commence à lire. Dans les deux classes, l'attention des élèves est orientée vers deux objets bien différents. Si M61 met bien en scène son propre comportement de lecteur avec l'utilisation ostensible du marque-page, le confort de sa posture de lecture, la relation à la fois respectueuse et intime qu'il instaure avec l'objet livre, M54 est au contraire très distante et maintient longuement un contact avec son outil de travail, le tableau, avant de commencer à lire. Et lorsqu'enfin elle tient le livre, c'est uniquement pour faire la lecture et non pour le manipuler, elle commence à lire dès qu'il est ouvert sans préparer l'écoute.

La sortie de lecture porte les mêmes caractéristiques : M61 referme doucement le livre et le tient couché sur ses genoux un doigt coincé dans la dernière page lue, geste qu'il maintiendra tout le long de l'échange qui va suivre. M54 ferme rapidement le livre et le pose sur le sol près d'elle, ce qui le fait disparaitre du champ d'observation des élèves.

Les élèves réagissent en relation avec cette gestualité. Un élève de c61 remarque un peu déçu « $\mathrm{Ah}$ / elle était pas longue » et dans c54 un garçon demande «maintenant/ on va lire les trois brigands » (il s'agit de l'album en cours d'étude et dont un extrait est écrit au tableau). La sortie de lecture en tant que changement de temporalité est immédiate pour les élèves de c54 alors que les élèves de c61 expriment justement leur ressenti sur cette temporalité particulière que le récit fait vivre et les prépare à l'échange qui suit la lecture.

On identifie ainsi dans la relation corporelle de l'enseignant avec le livre et avec le fait de le lire à ses élèves des éléments qui participent à l'interprétation des élèves sur le 
comportement lié à la lecture et leur permet d'entrer et sortir à la suite du maitre dans une temporalité du lecteur qui s'articule à celle du récit.

\subsection{Synthèse : des pratiques de LO fortement contrastées et une expertise professionnelle inégalement répartie entre Class+ et Class-}

37 Notre analyse nous permet de mettre en évidence un fort contraste entre Class+ et Classsur de nombreux points. Nous allons ici revenir sur les aspects plus remarquables de l'expertise professionnelle des maitres Class+ :

- Un haut degré de ritualisation: nos observations donnent à voir une ritualisation du temps réservé aux LO : cadre spatial réservé à cet usage, gestes qui marquent le début et la fin de lecture. Sur la dimension gestuelle, on observe de grandes régularités sur la manipulation du livre par les maitres, ce qui renforce l'idée d'une familiarité avec la lecture qui est culturellement codifiée et qui s'intègre dans une corporalité spécifique. Les gestes délicats démontrent la valeur attribuée à l'objet livre au moment de la lecture. L'entrée dans le texte et la sortie sont ritualisées, la fin de lecture est identique dans chaque classe, le livre est fermé lentement puis posé, première de couverture cachée sur les genoux. Lors des interactions, le livre est symboliquement placé au centre des discussions et la différenciation des interlocuteurs - maitre, livre et élèves - est clairement identifiable. L'identification des locuteurs est manifestée par des gestes de pointage vers les élèves et vers le livre.

39 - Un «climat » interactionnel favorisant l'investissement des élèves dans la tâche: plusieurs aspects sont à relever. D'abord des lectures adressées (regards de l'enseignant oscillant de manière régulière entre le texte et le regard des enfants, distribution équitable des regards de l'enseignant) : «Chacun peut se sentir destinataire personnel du récit, tout en étant soutenu dans son effort d'attention par la présence des autres embarqués dans un "ailleurs" commun. » (Chemla \& Dreyfus, 2012, p. 117) Cette manière de conserver le contact visuel semble favoriser la gestion de l'attention conjointe autour de l'objet livre.

Dans Class+, les LO mettent également à l'honneur ce que Chemla et Dreyfus (2012) nomment l'oral réflexif, sorte de continuum entre oral, écrit oralisé, texte. Dans ce contexte d'oral intermédiaire, la parole de l'enfant n'est pas un aléa toléré mais un élément constitutif de la séance, un aspect pensé de la stratégie pédagogique: l'oral réflexif ne peut fonctionner que si l'enseignant laisse suffisamment d'espace et de liberté de parole aux élèves. Ce scénario interactionnel spécifique rend possible, durant la lecture, la création d'un espace intime autour du texte par l'entremêlement permanent du lire, du voir et du parler.

41 - Un contrat de lecture clair : la LO s'inscrit dans un cadre institué qui spécifie à la fois les attentes des maitres et des élèves, rappelle les règles et l'objectif principal de la situation de LO : comprendre et interpréter des récits écrits. Cela passe par la mise en place d'une « médiation collective du parcours interprétatif » (Chemla \& Dreyfus, 2012).

42 Ce cadre et ce déroulé, parce qu'ils sont reconnus par les élèves, donnent du sens à la situation de LO, l'intègrent dans un tout cohérent dans lequel les élèves peuvent construire des repères stables. 
43 - Une médiation orale du texte d'une grande qualité : à l'instar des observations faites par Chemla et Dreyfus (2012), Class+ se caractérise par la grande qualité de la médiation orale de l'enseignant. Cette oralisation experte, marquée entre autres par des pauses rythmiques très nombreuses et ciblées, semble décisive pour l'investissement des élèves dans la tâche de compréhension/interprétation : elle permet la mise en place d'un " sas " de réflexivité autour du texte, d'un "espace du texte ", sorte de respiration partagée entre le texte, les illustrations, le maitre médiateur, les élèves. Cet espace fonctionne comme un déclencheur d'images mentales et de réflexions sur le texte lu.

- Une dimension littéraire forte favorisant la construction d'une posture interprétative : les enseignants de Class+ sont des experts dans le domaine de la littérature de jeunesse, leurs choix pédagogiques et didactiques illustrent cette expertise. Ce qu'ils visent, c'est avant tout la construction d'une posture interprétative chez leurs élèves. Pour ce faire, ils mettent en œuvre des stratégies de compréhension et d'évaluation du texte avec des allers/retours systématiques entre un point de vue personnel et culturel (distancié) (Dufays, 2011, p.229) et un recours à l'expérience personnelle de l'élève. Ce scénario interprétatif est également marqué pour une importante "gestion de l'incertitude" (Sensevy, 2009), l'élève étant considéré comme un "lecteur autorisé à interpréter et soucieux d'argumenter" (Chemla \& Dreyfus, 2012). On remarque enfin que, dans ce contexte, la dimension « plaisir » est assez fréquente et revendiquée : on lit pour se faire du bien, rire, comprendre le monde, être ensemble, etc. C'est l'idée de la littérature comme un « festin partagée » (Rouxel, 2005) qui est donnée à voir.

Comme nous avons essayé de l'illustrer dans ce texte, les pratiques des maitres dans Class- s'opposent pratiquement point par point à celles qui viennent d'être décrites ici.

\section{Conclusion}

$\mathrm{Au}$ moment de clore ce texte, revenons sur notre questionnement initial et sur les résultats les plus saillants de ce travail. Précisons d'abord à quel point le contraste entre Class+ et Class- nous a surprises : nous ne nous attendions pas à de telles différences de pratiques chez des maitres expérimentés ${ }^{6}$. On peut donc enseigner depuis de longues années et, comme les maitres de notre échantillon Class-, ne pas être en mesure de mettre en œuvre les gestes professionnels adéquats permettant l'institution de l'élève lecteur.

47 Nos deux échantillons se différencient à la fois sur la quantité de livres lus et la régularité de cette pratique sur la durée de l'année ${ }^{7}$. Les enfants ne sont donc pas égaux face aux LO, et tous ne bénéficient pas de cette immersion narrative qui constitue la matière première des apprentissages du lire-écrire.

Par ailleurs, cet article met en évidence un degré d'expertise très différencié dans les manières de mettre en œuvre la LO. Ainsi, la lecture de récits au CP ne semble pas être une pratique «naturelle » équitablement répartie chez l'ensemble des enseignants. Elle demeure au contraire un savoir-faire professionnel hétérogène encore largement à construire en formation autour des questions de « rapport à la lecture » de l'enseignant.

Mais au-delà de ce contraste, l'échantillon Class+ montre une homogénéité dans les manières de faire qui dessinent une pratique stabilisée maitrisée par une minorité d'enseignants. Nous faisons l'hypothèse que les enseignants, privés de directives claires dans les textes officiels concernant la lecture de récit, perçoivent de manière confuse 
l'enjeu de cette pratique. Aussi, lire des récits en classe peut avoir des effets plus ou moins grands sur le sens que l'élève donne à la lecture en tant que pratique sociale. Le format pédagogique ritualisé et expert que nous avons décrit dans cet article est nettement centré sur l'agir du lecteur. Les séances de Class+ fournissent aux élèves un cadre explicite sur ce qui attendu des élèves et ce que fait le maitre. Elles donnent aux élèves l'occasion de vivre une expérience puis de revenir dessus dans un jeu interactionnel qui s'apparente à un entretien d'explicitation (Vermersch, 1994).

Autre résultat qui mérite d'être souligné ici: les maitres experts, que nous pouvons nommer "passeurs de lecture", n'ont pas une pratique différenciée selon la zone d'éducation. Leur expertise peut s'exercer de la même façon avec tous les élèves, avec les mêmes exigences langagières, la même jubilation à partager des récits (Rouxel, 2005). Elle est fondée sur la richesse de l'étayage qui prend en charge la version interprétée du récit, une modélisation du lecteur dans ses postures corporelle, intellectuelle et cognitive et le déroulement de l'interaction. Nous pensons que les maitres experts, "passeurs de lecture », parviennent ainsi à configurer une séance pleine de sens et à mobiliser un type d'oral réflexif qui favorise l'engagement de l'élève.

Ainsi, le rituel de LO, pivot de l'acculturation à l'écrit en CP, permettrait d'instituer l'élève lecteur, quelle que soit son origine socioculturelle.

Cette étude est à poursuivre en creusant certains aspects que nous n'avons fait qu'évoquer ici : par exemple la question de l'engagement des élèves dans le rituel de LO, ou celle de l'oral réflexif et de la parole partagée dans les interactions autour du livre.

\section{BIBLIOGRAPHIE}

BisHop, Marie-France. (2012). La lecture des récits de fiction à l'école maternelle, histoire d'un genre professionnel. Le français aujourd'hui, 4(179), 113-126.

BoIron, Véronique. (2010). Lire des albums de littérature de jeunesse à l'école maternelle : quelques caractéristiques d'une expertise en actes. Repères, 42, 105-126.

BONNÉRY, Stéphane. (2012). Les outils sémiotiques et les dispositions sollicitées dans le Père Castor (Premières lectures) et la littérature enfantine depuis 1945 : sociologie historique des «lecteurs supposés ». Communication dans le cadre du séminaire « Paul Faucher (1898-1967) : L'édition au service de l'Éducation nouvelle », co-organisé par Dynadiv, Civiic, Circeft avec les Amis du Père Castor. BONNÉRY, Stéphane. (2014). Les livres et les manières de lire à l'école et dans les familles : réflexions à l'occasion de la parution de la liste officielle « maternelle ». Le français aujourd'hui, 185 (2), 47-57.

BONNÉRY, Stéphane. (dir.). (2015). Supports pédagogiques et inégalités scolaires : études sociologiques. Paris : La Dispute.

BONNÉRY, Stéphane \& JOIGNEAUX, Christophe. (2015). Des littératies familiales inégalement rentables scolairement. Le français aujourd'hui, 190(3), 23-34. 
BOURDIEU, Pierre. (1982). Les rites comme actes d'institution. Actes de la recherche en sciences sociales, $43,58-63$.

BouRHIS, Véronique. (2012). Situation de lecture en toute petite section : le rôle du paraverbal. Le français aujourd'hui, 179, 68-85.

BRUNER, Jérôme. (2005). Pourquoi nous racontons-nous des histoires? Le récit au fondement de la culture et de l'identité individuelle. Paris : Retz.

Chauveau, Gérard. (1997). Comment l'enfant devient lecteur. Pour une psychologie cognitive et culturelle de la lecture. Paris : Retz.

Chemla, Marie-Thérèse \& DReyfus, Martine. (2012). L'oral « intermédiaire » dans la lecture littéraire en cycle 2. Étude d'un moment de lecture feuilleton en GS : Samani, l'indien solitaire. Dans J.-C. Chabanne \& D. Bucheton (dir.), Parler et Écrire, pour penser, apprendre et se construire. L'écrit et l'oral réflexif. Paris: Presses universitaires de France.

DeZutTER, Olivier \& DufAys Jean-Louis. (2015). Enseigner et apprendre la lecture : des voyages dans l'espace et dans le temps. Repères, 51, 7-15.

DionNE Anne-Marie. (2013). Le plaisir de lire avec son enfant : un enjeu déterminant pour une pratique de littéracie aux multiples facettes. Forumlecture.ch, 3. <http://www.forumlecture.ch/ redaktionsbeitrag_2013_3.cfm, 2013>.

DUFAYS, Jean-Louis. (2011). Quel enseignement de la lecture et de la littérature à l'heure des « compétences »? Pratiques, 149-150, 227-248.

FRIER, Catherine (dir.). (2006). Passeurs de lecture. Lire ensemble à la maison et à l'école. Paris : Retz. GIASson, Jocelyne. (2011). La lecture. De la théorie à la pratique. Bruxelles : De Boeck.

Grossmann, Francis. (1996). Enfances de la lecture. Manières de faire, manières de lire à l'école maternelle. Berne : Peter Lang.

JORRO, Anne. (2006). L'agir professionnel de l'enseignant. Communication présentée au séminaire de recherche du Centre de recherche sur la formation - CNAM (fév. 2006), Paris.

MERRI, Maryvonne \& VANNIER, Marie-Paule. (2015). De l'affaiblissement au renouveau des rituels dans les institutions scolaires. Recherches en Éducation, 8 (hors-série), 3-13.

MiNISTÈRE DE L'ÉDUCATION NATIONALE. (2011). Grilles de références pour l'évaluation et la validation des compétences du socle commun au palier 1. DGESCO.

Ministère DE L'ÉDUCATION NATIONALE. (2015). Programmes d'enseignement du cycle des apprentissages fondamentaux (cycle 2), du cycle de consolidation (cycle 3) et du cycle des approfondissements (cycle 4). BOEN spécial n 10 du 19 novembre 2015.

Nonnon, Élisabeth \& GoIgoux, Roland. (2007). Travail de l'enseignant, travail de l'élève dans l'apprentissage initial de la lecture. Repères, 36, 5-36.

Olson, David R. (1999). L'Univers de l'écrit. Comment la culture écrite donne forme à la pensée. Paris : Retz.

PIETTE, Albert. (1997). Pour une anthropologie comparée des rituels contemporains. Terrain, 29, 139-150.

PiQuéE, Céline \& SENSEVY, Gérard. (2007). Lecture au cours préparatoire : une analyse empirique de l'influence des choix pédagogiques et didactiques. Repères, 36, 231-252.

RICœUR, Paul. (1983-1985). Temps et récit (3 vol.). Paris : Seuil. 
RouXEL, Annie. (2005). Lectures cursives : quel accompagnement? Paris : Delagrave- CRDP MidiPyrénées.

SENSEVY, Gérard. (2009). Étude d'un enseignement de la lecture au cours préparatoire : esquisse d'articulation de divers types d'analyse. Revue française de pédagogie, 168, 39-58. <https:// rfp.revues.org/1734>.

Tiré, Marianne, VadCAR, Anne, Ragano, Serge \& Bazile, Sandrine. (2015). Pour une approche objective des pratiques d'acculturation à l'écrit en classe de $\mathrm{CP}$ : essai de catégorisation et premières analyses. Repères, 52, 77-96.

TURNER, Victor W. (1969). Le Phénomène rituel. Structure et contre-structure. Paris : Presses universitaires de France.

VERMERSH, Pierre. (1994). L'entretien d'explicitation en formation initiale et continue. Paris : ESF.

Wulf, Christoph, Althans, Birgit, Audehm, Kathrin, Bausch, Constanze, GöHlich, Michael, Sting, Stephan, Tervooren, Anja, WAGner-Willi, Monika \& Zirfas, Jörg. (2004). Penser les pratiques sociales comme rituels. Ethnographie et genèse des communautés. Paris : L'Harmattan.

\section{NOTES}

1. Ces résultats sont à mettre en perspective des travaux de Jérôme Bruner, qui soulignent l'importance capitale du récit dans l'élaboration de la pensée humaine (2005) ou ceux de Paul Ricœur (1983-1985) qui lient le développement de la conscience à celle d'une identité narrative. L'aptitude au récit, matrice originelle qui comporte en son sein toutes les autres formes discursives, serait ainsi une composante de notre humanité, développée dans le cadre des interactions éducatives (familiales, scolaires).

2. Bonnéry et Joigneaux (2015) montrent par exemple l'existence de trois modèles familiaux: lectures « oralisation » ou « dirigées » des familles populaires à faible capital culturel ou scolaire et «indiciaires» des familles à fort capital culturel ou scolaire. Cet entrainement indiciaire permet la construction de «techniques intellectuelles consistant à suspendre régulièrement le flux des lectures pour prendre le temps du recul par rapport à ce qui a été lu ou vu, et se donner le moyen de tisser des liens tacites dans les textes. Ces techniques et activités intellectuelles sont, selon les auteurs, celles qui sous-tendent les manières de lire les plus rentables durant la suite de la scolarité.

3. Par «choix didactiques", nous entendons, à l'instar de Piquée et Sensevy (2007), « les choix relatifs aux supports utilisés en classe et les contenus enseignés» (p.231). Les "choix pédagogiques » quant à eux renvoient davantage à la manière spécifique dont sont enseignés ces contenus.

4. Il s'agit d'une approche écologique qui « consiste à étudier des classes ordinaires telles qu'elles fonctionnent quotidiennement, sans manipulation du contexte par le chercheur, c'est-à-dire sans modification de l'action habituelle des enseignants" (Rapport Lire-Écrire au CP consultable à l'adresse <http://ife.ens-lyon.fr/ife/recherche/lire-ecrire/rapport/rapport>).

5. L'observation est réduite à seulement 4 classes de Class+ et 4 de Class-. Les films ne permettaient pas toujours de bien observer la gestualité du maitre.

6. Rappelons que tous les enseignants du corpus IFÉ sont des maitres expérimentés. Compte-tenu de nos objectifs (mesurer l'effet des pratiques sur les performances des élèves), ce choix devait nous permettre d'éviter le biais de pratiques débutantes, encore peu stabilisées et difficiles à interpréter. 
7. Les classes 52 et 93 n'ont pas fait de lecture offerte d'après les questionnaires, la classe 13 ne déclare sur les questionnaires que deux lectures offertes (sur huit semaines de classes).

\section{RÉSUMÉS}

Ce texte a pour objectif de décrire et d'analyser la médiation au récit écrit en classe de Cours préparatoire. Nous faisons ici l'hypothèse que cette médiation relève d'une expertise professionnelle qui se décline à travers des gestes précis restant à qualifier et qu'elle s'inscrit dans le cadre d'une ritualisation à l'interface de la sphère scolaire, sociale et culturelle. La démarche méthodologique choisie permet de croiser données quantitatives et données qualitatives, observations de classes et discours sur les pratiques et amène à mesurer l'impact des choix didactiques de l'enseignant dans ce domaine.

This article intends to describe and analyze the mediation of narrative reading in first-grade classes. We make the hypothesis that this mediation comes from a professional expertise that is declined through specific actions still to be qualified and that it is part of a ritualization at the interface of the school, social sphere and cultural sphere. The selected methodological approach allows crossing quantitative data with qualitative data, classes sighting and discourse on practices. It leads to measure the impact of the didactic choices of the teacher in this field.

\section{INDEX}

Mots-clés : didactique de la lecture $\mathrm{CP}$, lecture de récits, agir enseignant Keywords : reading didactic, narrative reading, teaching performance

\section{AUTEURS}

\section{ANNE VADCAR}

Université Grenoble Alpes, LIDILEM

\section{CATHERINE FRIER}

Université Grenoble Alpes, LIDILEM 